\title{
The bottom line
}

\author{
Our understanding of actinide chemistry lags behind that of the rest of the periodic table. A collection of \\ articles in this issue highlights recent progress featuring uranium( $\mathrm{VI}$ ) dianions bearing four U-N multiple \\ bonds, berkelium(IV) stabilized in solution and delocalization of $5 f$ electrons in a plutonium material.
}

Of all of the series within the periodic table, that of the actinides is the one we understand the least. This partly arises from their nuclear instability: they are all radioactive, which brings with it experimental difficulties and health hazards of various extents depending on the nature and rate of their decay. Transuranium elements are handled in dedicated facilities, and planning experiments involves considering emission from not just their own radioactive decay, but also that of their daughter elements. Furthermore, although early actinides such as thorium and uranium are relatively abundant, others suffer from limited availability and costly production processes. Beyond such practical challenges, another aspect that has hampered our ability to accurately predict the behaviour of actinides is that relativistic effects significantly affect their electronic structure.

A crucial line of enquiry with actinides involves understanding the degree of localization of the $5 f$ orbitals and the extent to which they can engage in covalent bonding. This not only allows the principles that underscore their chemical behaviour to be determined, but is also critical for practical applications such as nuclear-waste management and environmental remediation. Actinides are difficult to separate, both from each other and from their $f$-block neighbour lanthanides, so these processes must rely on subtle bonding differences.

An understanding of actinide bonding and its manipulation is also central to other research directions currently being explored, which include cancer therapy (through their emission of a particles), molecular magnetism and actinide-mediated reactivity; for example, small-molecule activation by uranium and thorium catalysts ${ }^{1}$.

One avenue of investigation is to probe actinide-ligand complexes with multiplebonding character. This area has recently seen exciting developments, including the isolation of terminal oxo-, nitrido- and carbene-containing complexes, actinide chalcogenides ${ }^{2}$ and analogues of the highly-valent actinyl ions $\left(\left[\mathrm{AnO}_{2}\right]^{2+}\right)-\mathrm{a}$ ubiquitous motif that is characterized by its two strong trans-An-O multiple bonds. Nitrogen analogues of uranyl have attracted particular attention. Suzanne Bart and co-workers recently prepared a series of uranium compounds featuring U-N multiple bonds by using a redox-flexible pyridine(diimine) ligand to destabilize the strong trans bonds through heavy $\pi$ donation $^{3}$. This led to the formation of uranium mono-, bis- and tris(imido) products, depending on the extent of the ligand reduction. Now, as described on page 850 of this issue, the researchers have extended this approach to prepare tetrakis(imido)uranium(vi) dianions, thus featuring four U-N multiple bonds.

In an Interview on page 832,

Suzanne Bart shares what attracted her to actinide chemistry and what she finds most challenging and rewarding.

Besides their versatile coordination chemistry, the actinides also show rich redox behaviour. The earlier actinides can adopt a number of oxidation states in solution, but the heavier ones are less inclined to do so. Nevertheless, because of its half-filled $5 f^{7}$ electronic configuration, berkelium is an anomaly and has been predicted to be able to exhibit both +III and +IV oxidation states in solution. It is, however, a particularly difficult element to study because ${ }^{249} \mathrm{Bk}$ - the only berkelium isotope available in appreciable quantities - has a relatively short half-life of 330 days, and its compounds degrade rapidly. There have been some successes, with Bk(III) having previously been shown to be oxidizable to its +IV state, but only for short periods of time and under drastic conditions. Overcoming the experimental challenges that come with working with it, Gauthier Deblonde and co-workers have now succeeded in stabilizing $\mathrm{Bk}$ (IV) in solution under mild conditions, as described on page 843 . They do so through its strong complexation with a synthetic octadentate siderophore analogue - previously proven a good chelator for the stabilization of +III and +IV states, with a strong preference for the latter.

If that's not enough berkelium for you, Andreas Trabesinger relays its story in an 'In Your Element' essay on page 924, from its challenging generation, to its main use as a target for the synthesis of ever heavier elements, and on to very recent explorations of its chemistry. He highlights how, only last year, a major breakthrough was made when $\mathrm{Bk}(\mathrm{III})$ complexes were isolated and characterized, including by single-crystal $\mathrm{X}$-ray diffraction ${ }^{4}$. The research showed how spin-orbit coupling contributed significantly to berkelium's ground state.

Beyond mononuclear species, an interesting feature of multinuclear compounds is that electron transfer may occur between different metal centres possessing different oxidation states. Such electronic delocalization - referred to as intervalence charge-transfer (IVCT) - is largely unexplored in actinides. However, given that it relies on spatial overlap between metal and ligand orbitals, it is wellestablished for various $d$-block transitionmetal complexes, clusters and materials. The degree of the electronic delocalization between the metal centres is classified by the Robin-Day system. In class I compounds the oxidation state at each metal is localized, whereas in their class III counterparts complete delocalization occurs so that the metal centres are equivalent; class II compounds show intermediate behaviour. The $5 f$ and nearly degenerate $6 d$ orbitals of actinides are, in principle, amenable to interacting with ligand orbitals, and this is exactly what Thomas Albrecht-Schmitt and co-workers describe on page 856 - a mixedvalent plutonium solid-state compound that shows incipient class II behaviour. It exhibits distinct $\mathrm{Pu}(\mathrm{III})$ and $\mathrm{Pu}(\mathrm{IV})$ sites, yet experimental and computational evidence point to the delocalization of its $5 f$ electrons.

These articles showcase some of the recent insights gained into the elements at the bottom of the periodic table, bringing new understanding that has both fundamental and practical importance. Such endeavours are increasingly supported by technical progress, both experimentally and computationally, and thus we expect a clearer picture of the bonding, reactivity and electronic structure of these elements to continue to emerge.

\footnotetext{
References

1. Ephritikhine, M. Organometallics 32, 2464-2488 (2013).

2. Hayton, T. W. Chem. Commun. 49, 2956-2973 (2013).

3. Anderson, N. H. et al. Nat. Chem. 6, 919-926 (2014).

4. Silver, M. A. et al. Science 353, aaf3762 (2016).
} 